\title{
DEVELOPING ASSESSMENT LITERACY
}

Dr Lynda Taylor 


\section{Overview}

- 1 : Introduction to Assessment Literacy

- 2: Principles and Practice of Language Assessment 


\section{Introduction to Assessment Literacy}




\section{Definitions of 'literacy'?}

-What do we mean/understand by the word 'literacy'?

lit.er.a.cy $\mathrm{n}$.

1. The condition or quality of being literate, especially the ability to read and write.

2. The condition or quality of being

knowledgeable in a particular subject or field: cultural literacy; academic literacy 


\section{Definitions of 'academic literacy'?}

- the ability to read and write effectively within the college context in order to proceed from one level to another

- the ability to read and write within the academic context with independence, understanding and a level of engagement with learning

- familiarity with a variety of discourses, each with their own conventions

- familiarity with the methods of inquiry of specific disciplines 


\section{An age of literacy (or literacies)?}

- 'academic literacy/ies'

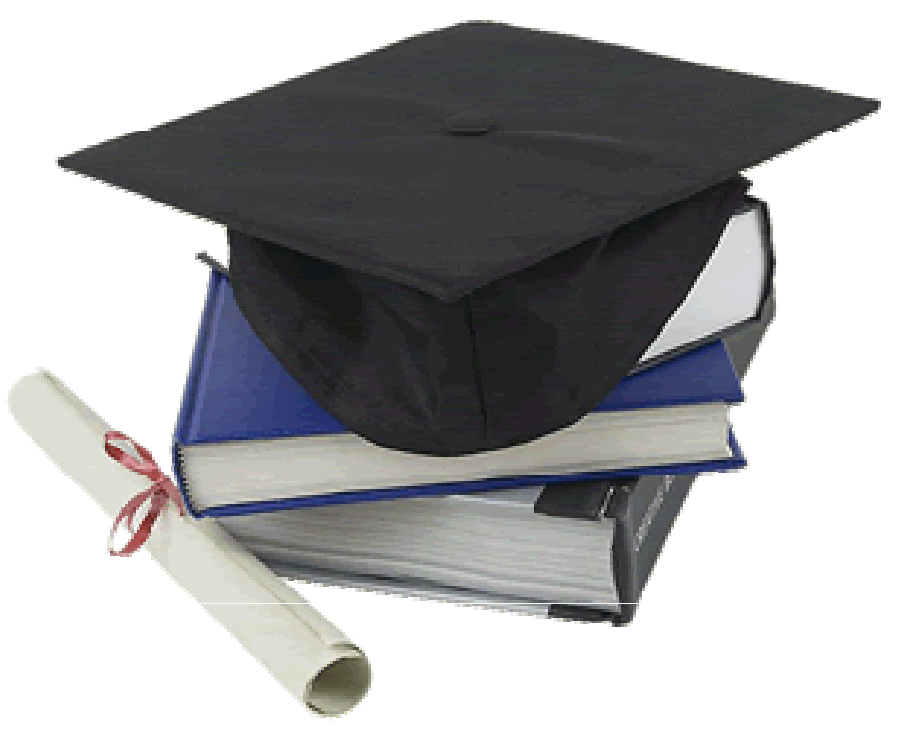

- 'computer literacy'

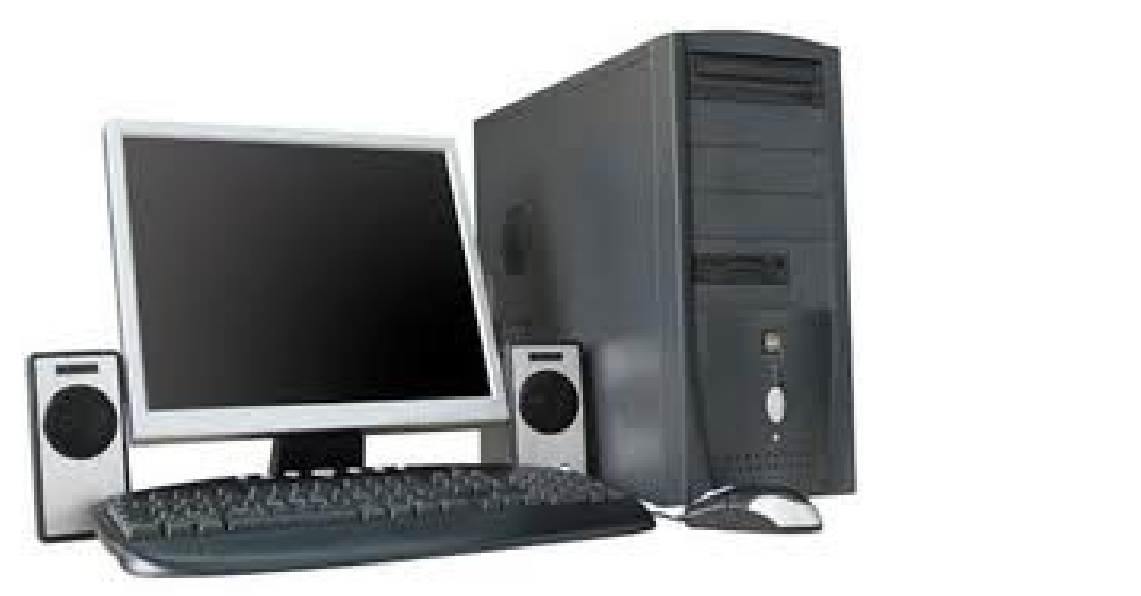




\section{An age of literacy (or literacies)?}

- 'civic literacy'

- 'risk literacy'
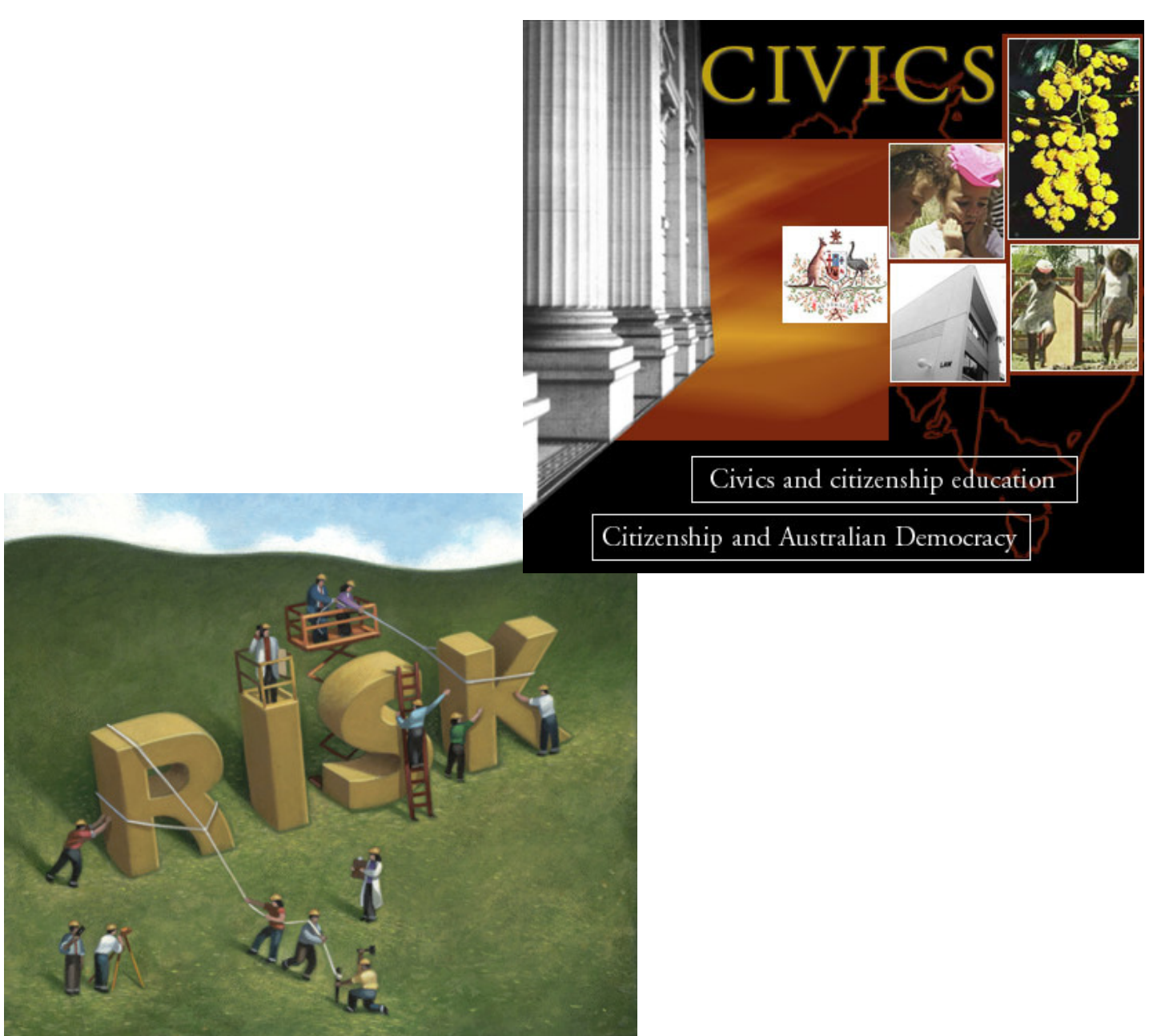
What do we understand by 'risk literacy'?

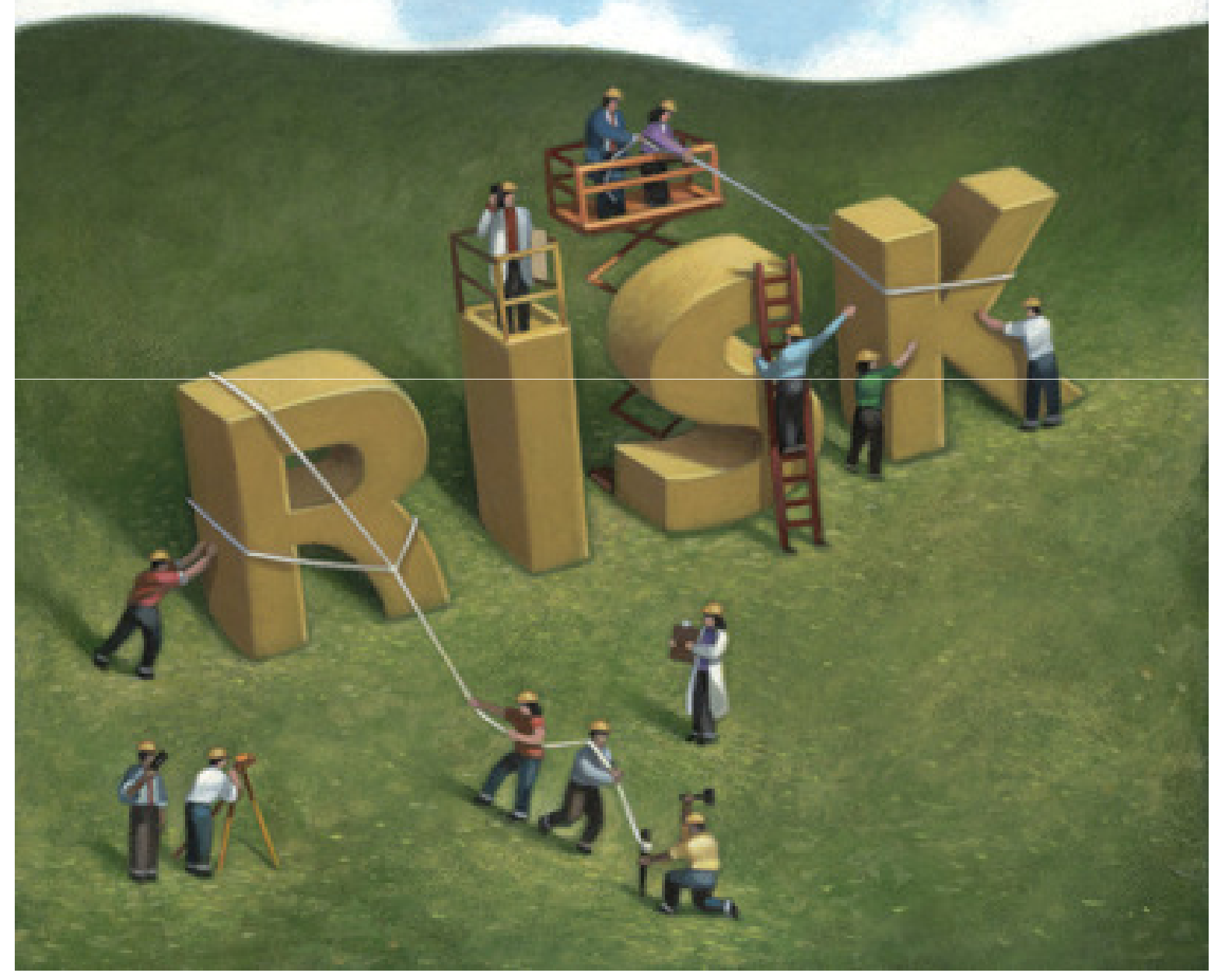




\section{'Risk literacy' has been defined as...}

- 'a basic grasp of statistics and probability'

- 'statistical skills to make sensible life decisions'

But why might it be useful/important? 


\section{Prof David Spiegelhalter (2009)}

\section{Risk literacy:}

- 'critical to choices about health, money and even education'

- 'as the internet transforms access to information, it is becoming more important than ever to teach people how best to interpret data' 


\section{Prof David Spiegelhalter (2009)}

- '...we should essentially be teaching the ability to deconstruct the latest media story about a cancer risk or a wonder drug, so people can work out what it means. Really, that should be part of everyone's language...' 


\section{Some key words/concepts so far...}

- ability (to read + write)

- 'basic grasp'

- knowledge

- 'access to information'

- skills

- subject/field/discipline

- discourses

- conventions/methods

- understanding

- level of engagement

- 'teach people how best to interpret data'

- 'within a context'

- 'critical to choices'

- 'skills to make sensible life decisions'

- 'ability to deconstruct'

- 'work out what it means'

- 'part of everyone's language' 


\section{'Assessment literacy' ...}

- 'a basic grasp of numbers and measurement'

- 'interpretation skills to make sensible life decisions'

But why is it necessary or important?

And who needs it? 


\section{The background (1)}

- The growth of language testing and assessment worldwide

- public examinations systems at national level

- primary, secondary and tertiary levels

- alignment of testing systems with external frameworks, e.g. CEFR, CLB

- measures of accountability

- other socio-economic drivers, e.g. immigration, employment 


\section{The background (2)}

- Growing numbers of people involved in language testing and assessment

- test takers

- teachers and teacher trainers

- university staff, e.g. tutors \& admissions officers

- government agencies and bureaucrats

- policymakers

- general public 
'Language testing has become big business...'

(Spolsky 2008, p 297) 


\section{But...}

... how 'assessment literate' are all those people nowadays involved in language testing and assessment across the many different contexts identified? 


\section{Assessment literacy involves...}

- an understanding of the principles of sound assessment

- the know-how required to assess learners effectively and maximise learning

- the ability to identify and evaluate appropriate assessments for specific purposes

- the ability to analyse empirical data to improve one's own instructional and assessment practices

- the knowledge and understanding to interpret and apply assessment results in appropriate ways

- the wisdom to be able to integrate assessment and its outcomes into the overall pedagogic/decisionmaking process 


\section{Assessment literacy involves...}

\section{Skills}

$+$

Knowledge

$+$

Principles 


\section{Attitudes to tests, test scores and their experience of assessment?}

- Students

- Language teachers

- Educational boards

- Politicians and policy-makers 
'... an oversimplified view of the ease of producing meaningful measurement is held by the general public and educational administrators alike ...'

(Spolsky 2008, p 300) 


\section{Barriers to assessment literacy?}

- Negative emotions/fear of numbers?

- A very specialised/technical field? (professionalisation of language testing)

- Too time-consuming?

- Insufficient resources? 


\section{Principles and practice of language assessment}




\section{Aim of language assessment}

- To measure a latent (largely unobservable) trait

- But how?

- Aim = to make inferences about an individual's language ability

- based on observable behaviour(s)

- to which 'test scores' are attached

- The process of 'score interpretation' involves giving meaning to numbers 


\section{The over-arching principle of test 'utility'/'usefulness'}

- Test purpose must be clearly defined and understood

- Testing approach must be 'fit for purpose'

- There must be an appropriate balance of 'essential test qualities' (such as validity and reliability) 


\section{Defining sub-principles of usefulness}

Usefulness $=$ Reliability

+ Construct validity

+ Authenticity

+ Interactiveness

+ Impact

+ Practicality

(Bachman and Palmer 1996) 


\section{Bachman and Palmer (1996)}

- Characteristics of the language use task and situation

- Characteristics of the test task and situation

- LANGUAge USE

- LANGUAGE TEST PERFORMANCE

- Characteristics of the language user

- Topical knowledge

- Affective schemata

- Language ability

- Characteristics of the test taker

- Topical knowledge

- Affective schemata

- Language ability 


\section{Weir (2005)}

- A socio cognitive framework for test development and validation, embracing:

- Test taker characteristics

- Cognitive validity

- Context validity

- Scoring validity

- Consequential validity

- Criterion-related validity 
Testing/assessment cultures can shape both principles and practice

- The role of historical and social context

- USA tradition

- psychometric focus

- 'objective' test formats - MCQ

- UK tradition

- interface with pedagogy

- focus on validity - task-based 


\section{Some current issues for debate in the assessment community}

- Washback and impact

- Accountability and ethical testing

- Technological developments

- Developments in linguistics 


\section{Language testing/assessment}

- A historical, educational, sociological, technological phenomenon - a good example of a 'complex system'

- An 'inherently interdisciplinary venture'

- Assessment 'serves other disciplines, and vice versa' and 'the boundaries between theory and application are increasingly blurred' 


\section{This 2-week course is designed to help develop your assessment literacy}

- ability

- knowledge

- skills

- subject/field/discipline

- discourses

- conventions/methods

- understanding

- level of engagement
- 'basic grasp'

- 'access to information'

- 'teach how best to interpret data'

- 'within a context'

- 'critical to choices'

- 'skills to make sensible decisions'

- 'ability to deconstruct'

- 'work out what it means'

- 'part of the language' 


\section{Enjoy your time here at CRELLA}

developing your assessment literacy!

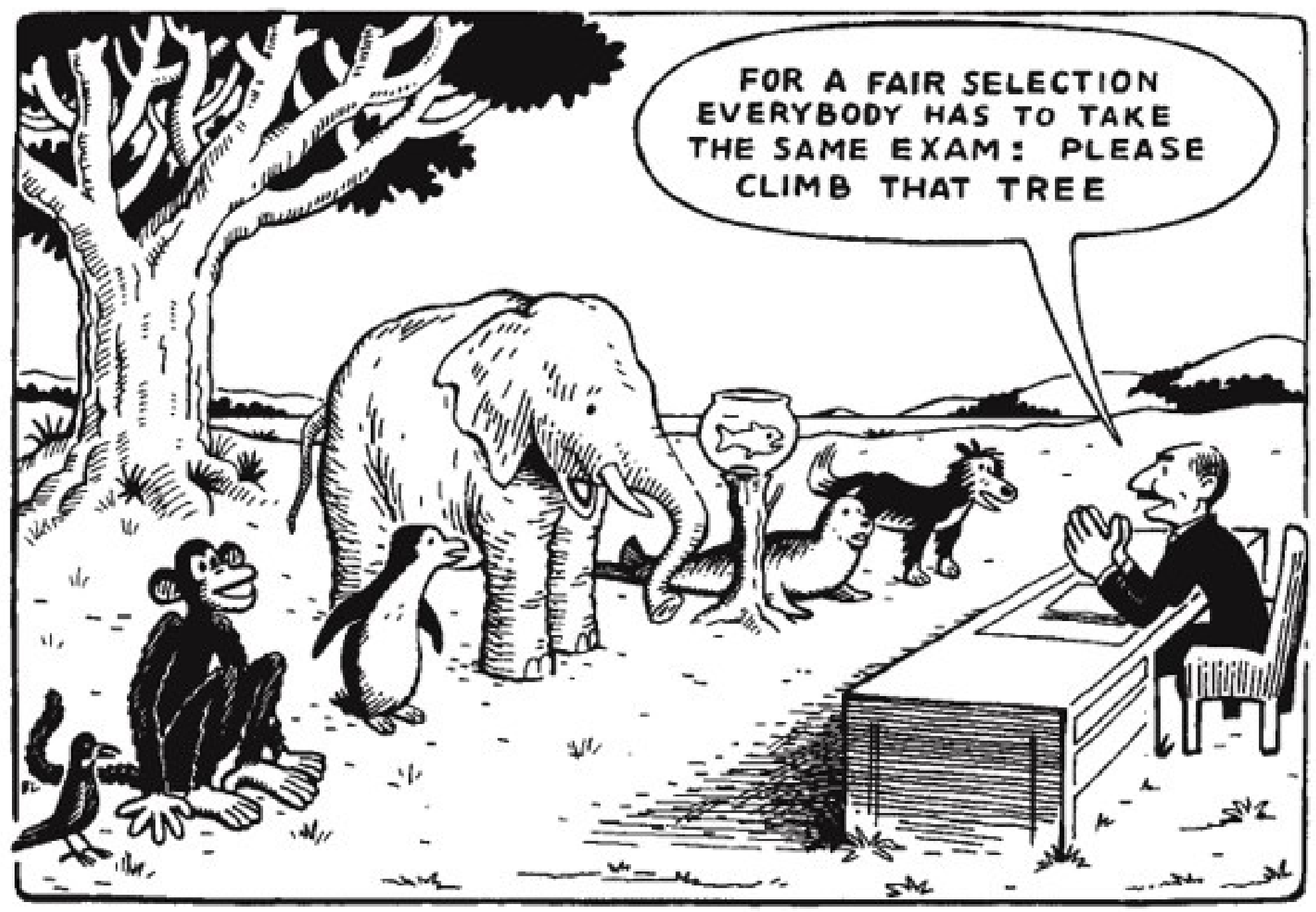

